\title{
OPERATOR-VALUED MULTIPLIER THEOREMS CHARACTERIZING HILBERT SPACES
}

\author{
WOLFGANG ARENDT and SHANGQUAN BU
}

(Received 22 May 2002; revised 4 June 2003)

Communicated by G. Willis

\begin{abstract}
We show that the operator-valued Marcinkiewicz and Mikhlin Fourier multiplier theorem are valid if and only if the underlying Banach space is isomorphic to a Hilbert space.

2000 Mathematics subject classification: primary 42A45, 42C $99,46 \mathrm{Cl} 5,47 \mathrm{~A} 56$.

Keywords and phrases: Operator-valued Fourier multiplier, Hilbert space and Rademacher boundedness.
\end{abstract}

\section{Introduction}

Mikhlin's multiplier theorem is of great importance in analysis. It says that a bounded function $m \in C^{l}(\mathbb{R} \backslash\{0\})$ such that $t m^{\prime}(t)$ is bounded, defines an $L^{p}(\mathbb{R})$-multiplier for $1<p<\infty$. In the context of partial differential equations vector-valued spaces $L^{p}(\mathbb{R} ; X)$ occur in a natural way, where $X$ is a Banach space. Thus the function $m$ should take its values in $\mathscr{L}(X)$. Our aim is to show that Mikhlin's multiplier theorem does hold for such operator-valued functions if and only if $X$ is isomorphic to a Hilbert space.

The phenomenon that operator-valued versions of certain classical multiplier theorems are only valid in Hilbert spaces was first observed by Pisier (unpublished) as a consequence of Kwapien's deep characterization of Hilbert spaces. More recently, new versions of operator-valued multiplier theorems turned out to be most useful in the theory of evolution equations (see the references and comments below) and it

This research is part of the DFG-project: "Regularität und Asymptotik für elliptische und parabolische Probleme". The second author is supported by the Alexander-von-Humboldt Foundation, the NSF of China and the Excellent Young Teacher Program of MOE, P.R.C.

(C) 2004 Australian Mathematical Society 1446-7887/04 \$A2.00+0.00 
seems to us that it is important to elaborate in some detail why the classical result merely holds on Hilbert spaces.

In another context, it helps to impose a Mikhlin's condition of order $k$

$$
m \in C^{k}(\mathbb{R} \backslash\{0\} ; \mathscr{L}(X)), \quad \sup _{t \in \mathbb{R} \backslash\{0\}, 0 \leq l \leq k}\left\|t^{l} m^{(l)}(t)\right\|<\infty
$$

In fact, Amann [1] discovered that if $m$ satisfies (1) with $k=2$, then $m$ is a multiplier for Besov spaces and in particular for the space $C^{\theta}(\mathbb{R} ; X), 0<\theta<1$ (see also [2] and [11]). We show here that imposing higher order Mikhlin's conditions does not help in the context of operator-valued $L^{p}$-multipliers.

We also consider the groups $\pi$ and $\mathbb{Z}$ instead of $\mathbb{R}$. In fact, the case $\mathbb{T}$ corresponds to Marcinkiewicz's classical theorem and its operator-valued version is already treated in [3] for the order-1-case.

Now we would like to comment on the new vector-valued multiplier theorems which were found recently. It were Berkson-Gillespie [4] who introduced the notion of $R$-boundedness (after implicit use of Bourgain [6]). They use $R$ as an abbreviation for Riesz, but in many subsequent papers people seem think rather of Rademacher or 'Randomized' because the definition involves Rademacher functions. A multiplier theorem of Marcinkiewicz type was established by Clément-de Pagter-SukochevWitvliet [8] for multipliers of the form $m(t) I$ ( $I$ is the identity operator) clarifying the role of $R$-boundedness. Then Weis [18] established Mikhlin's theorem for operator-valued functions (without restriction) replacing boundedness by the stronger condition of $R$-boundedness. Then in [3] the corresponding periodic theorem (that is, Marcinkiewicz's theorem) was proved on the basis of results in [8]. Strkalj and Weis [17] gave an $R$-version of the variational version of the Marcinkiewicz theorem. Further important contributions were given by Clément-Prüss [9], Denk-Hieber-Prüss [10], and Girardi-Weis [11].

\section{Periodic multipliers}

Let us first recall some notions. Let $X$ be a Banach space. Denote by $r_{j}$ the $j$-th Rademacher function on $[0,1]$. For $x \in X$, we denote by $r_{j} \otimes x$ the vector-valued function $t \mapsto r_{j}(t) x$. Let $Y$ be another Banach space. We denote by $\mathscr{L}(X, Y)$ the set of all bounded linear operators from $X$ to $Y$. If $X=Y$ we will denote $\mathscr{L}(X, Y)$ simply by $\mathscr{L}(X)$. A family $\mathbf{T} \subset \mathscr{L}(X, Y)$ is called $R$-bounded for fome $q \in[1, \infty)$ there exists a constant $c_{q} \geq 0$ such that

$$
\left\|\sum_{j=1}^{n} r_{j} \otimes T_{j} x_{j}\right\|_{L^{q}(0,1 ; Y)} \leq c_{q}\left\|\sum_{j=1}^{n} r_{j} \otimes x_{j}\right\|_{L^{q}(0,1 ; X)}
$$


for all $T_{1}, \ldots, T_{n} \in \mathbf{T}, x_{1}, \ldots, x_{n} \in X$ and $n \in \mathbb{N}$. By Kahane's inequality [14, Theorem 1.e.13] if such constant $c_{q}$ exists for some $q \in[1, \infty)$, then it also exists for each $q \in[1, \infty)$.

It is known that $R$-boundedness is strictly stronger than boundedness in operator norm unless the underlying Banach space is isomorphic to a Hilbert space. More precisely, each bounded subset in $\mathscr{L}(X, Y)$ is $R$-bounded if and only if $X$ is of cotype 2 and $Y$ is of type 2 (see [3, Proposition 1.13]). In particular, by a result of Kwapien [14, pages 73-74], each bounded subset in $\mathscr{L}(X)$ is $R$-bounded if and only if $X$ is isomorphic to a Hilbert space.

For $1 \leq p<\infty$, consider the Banach space $L^{p}(0,2 \pi ; X)$ with norm $\|f\|_{p}:=$ $\left(\int_{0}^{2 \pi}\|f(t)\|^{p} d t\right)^{1 / p}$. For $f \in L^{p}(0,2 \pi ; X)$ we denote by

$$
\hat{f}(k):=\frac{1}{2 \pi} \int_{0}^{2 \pi} e^{-i k t} f(t) d t
$$

the $k$-th Fourier coefficient of $f$, where $k \in \mathbb{Z}$. For $k \in \mathbb{Z}, x \in X$ we let $e_{k}(t)=e^{i k t}$ and $\left(e_{k} \otimes x\right)(t)=e_{k}(t) x \quad(t \in \mathbb{R})$. A function $f \in L^{p}(0,2 \pi ; X)$ is called a trigonometric polynomial if $f$ is given by $f=\sum_{k \in \mathbb{Z}} e_{k} \otimes x_{k}$, where $x_{k} \in X$ is 0 for all but finitely many $k \in \mathbb{Z}$.

Let $M=\left(M_{k}\right)_{k \in \mathbb{Z}} \subset \mathscr{L}(X, Y)$ be a sequence and let $1 \leq p, q<\infty$. We say that $\left(M_{k}\right)_{k \in \mathbb{Z}}$ is a periodic $L^{p}-L^{q}$-Fourier multiplier if there exists a constant $C>0$ such that

$$
\left\|\sum_{k \in \mathbb{Z}} e_{k} \otimes M_{k} x_{k}\right\|_{L^{q}(0,2 \pi ; Y)} \leq C\left\|\sum_{k \in \mathbb{Z}} e_{k} \otimes x_{k}\right\|_{L^{p}(0,2 \pi ; X)}
$$

for all $X$-valued trigonometric polynomials $\sum_{k \in \mathbb{Z}} e_{k} \otimes x_{k}$. In this case, there exists a unique operator $M \in \mathscr{L}\left(L^{p}(0,2 \pi ; X), L^{q}(0,2 \pi ; Y)\right)$ such that $(M f)^{\prime}(k)=M_{k} \hat{f}(k)$ for $k \in \mathbb{Z}$ [3]. When $p=q$, we say simply that $\left(M_{k}\right)_{k \in \mathbb{Z}}$ is a periodic $L^{p}$-Fourier multiplier. For $k \in \mathbb{Z}$, we let $\left(\Delta^{1} M\right)(k)=M_{k+1}-M_{k}$ and $\left(\Delta^{m} M\right)(k)=\left(\Delta^{1}\left(\Delta^{m-1} M\right)\right)(k)$ for $m \geq 2$. Notice that $\Delta^{m} M$ is a discrete analogue of the $m$-th derivative of $M$.

The classical Marcinkiewicz Fourier multiplier theorem has been extended to the operator-valued case in the following way: let $X$ and $Y$ be UMD spaces and let $\left(M_{k}\right)_{k \in \mathbb{Z}} \subset \mathscr{L}(X, Y)$; if both $\left\{M_{k}: k \in \mathbb{Z}\right\}$ and $\left\{k\left(M_{k+1}-M_{k}\right): k \in \mathbb{Z}\right\}$ are $R$-bounded, then $\left(M_{k}\right)_{k \in Z}$ defines a periodic $L^{p}$-Fourier multiplier for each $1<p<\infty$ [3]. Indeed, $\left(M_{k}\right)_{k \in Z}$ is a periodic $L^{p}-L^{q}$-Fourier multiplier whenever $1 \leq q \leq p<\infty$.

We will need the following inequality of Pisier [15]. Let $1 \leq p<\infty$ and let $\Lambda=\left\{n_{k}: k \in N\right\} \subset \mathbb{Z}$ be a Sidon subset [16, page 120]. Then there exists $C>0$ such that for any Banach space $X$ and for any finite sequence $\left(y_{k}\right)_{1 \leq k \leq N}$ of $X$, we have

$$
C^{-1}\left\|\sum_{k} r_{k} \otimes y_{k}\right\|_{2} \leq\left\|\sum_{k} e_{n_{k}} \otimes y_{k}\right\|_{p} \leq C\left\|\sum_{k} r_{k} \otimes y_{k}\right\|_{2} \text {. }
$$


Note that if $\lambda>1$, then any subset $\left\{n_{k}: k \in \mathbb{N}\right\}$ satisfying $n_{k+1} / n_{k} \geq \lambda(k \in \mathbb{N})$ is a Sidon subset of $\mathbb{Z}$ [16, page 127].

The following result shows that one cannot replace $R$-boundedness in the operatorvalued Marcinkiewicz theorem above by boundedness in operator norm unless the underlying Banach space is isomorphic to a Hilbert space.

THEOREM 1. Let $X$ be a Banach space. Then the following assertions are equivalent:

(i) $X$ is isomorphic to a Hilbert space.

(ii) For some $1 \leq q<p<\infty$, each sequence $\left(M_{k}\right)_{k \in \mathbb{Z}} \subset \mathscr{L}(X)$ satisfying

(a) $\sup _{k \in \mathbf{Z}}\left\|M_{k}\right\|<\infty$,

(b) $\sup _{k \in \mathbf{Z}}\left\|k^{l}\left(\Delta^{l} M\right)(k)\right\|<\infty$ for $l \in \mathbb{N}$,

(c) $M_{k}=0$ for $k \leq 0$,

is a periodic $L^{p}$ - $L^{q}$-Fourier multiplier.

(iii) For all $1<p<\infty$, each sequence $\left(M_{k}\right)_{k \in \mathbb{Z}} \subset \mathscr{L}(X)$ satisfying

(a) $\sup _{k \in \mathbb{Z}}\left\|M_{k}\right\|<\infty$,

(b) $\sup _{k \in \mathbb{Z}}\left\|k\left(\Delta^{1} M\right)(k)\right\|<\infty$,

is a periodic $L^{p}$-Fourier multiplier.

REMARK 2. For $l=1$, the condition formulated in (iii) is the classical condition considered by Marcinkiewicz in the scalar case. For arbitrary $l \in \mathbb{N}$ we therefore speak of the Marcinkiewicz condition of order $l$. For $p=q$ and $l=1$, Theorem 1 has been proved in [3, Proposition 1.17.]. However, a more refined choice of test functions is needed here for the general case. The motivation to consider $l>1$ stems from the results on Fourier multipliers for spaces of Hölder continuous functions where, indeed, the Marcinkiewicz condition of order 2 suffices (see [2] and also the Concluding Remarks at the end of this article). Theorem 1 shows that this is not the case in the $L^{p}$-context even if we consider weaker multipliers by allowing $q<p$. This has also been done by Kalton-Lancien in the context of maximal regularity for Cauchy problems [13] (see also the Concluding Remarks 5 (b) below).

Proof. (i) $\Rightarrow$ (iii). Assume that $X$ is isomorphic to a Hilbert space, then considering an orthonormal basis one easily verifies that each bounded subset in $\mathscr{L}(X)$ is actually $R$-bounded, so the result follows from the operator-valued Marcinkiewicz Fourier multiplier theorem in [3].

(iii) $\Rightarrow$ (ii) is trivial.

(ii) $\Rightarrow$ (i). Assume that for some $1 \leq q<p<\infty$, each sequence $\left(M_{k}\right)_{k \in \mathbb{Z}} \subset \mathscr{L}(X)$ satisfying $\sup _{k \in \mathbf{Z}}\left\|M_{k}\right\|<\infty, \sup _{k \in \mathbb{Z}}\left\|k^{l}\left(\Delta^{l} M\right)(k)\right\|<\infty$ for $l \in \mathbb{N}$ and $M_{k}=0$ for $k \leq 0$, is a periodic $L^{p}-L^{q}$-Fourier multiplier. Let $N=\left(N_{k}\right)_{k \in \mathbb{N}} \subset \mathscr{L}(X)$ be a bounded sequence. 
Let $\mathscr{S}(\mathbb{R})$ be the Schwartz space. Let $\phi_{1} \in \mathscr{S}(\mathbb{R})$ be such that $\operatorname{supp}\left(\phi_{1}\right) \subset[2,4]$ and $\phi_{1}(3)=1$. For $n \geq 1$, we let $h_{n}=2^{2 n-2}$. Define $\phi_{n}=\phi_{1}\left(\cdot / h_{n}\right)$. Then $\operatorname{supp}\left(\phi_{n}\right) \subset\left[2 h_{n}, 4 h_{n}\right]$ and $\phi_{n}\left(3 h_{n}\right)=1$. Let $\phi: \mathbb{R} \rightarrow \mathscr{L}(X)$ be defined by

$$
\phi(t)= \begin{cases}\phi_{n}(t) N_{n} & \text { if } 2 h_{n} \leq t \leq 4 h_{n} \text { for some } n \geq 1 \\ 0 & \text { otherwise. }\end{cases}
$$

Let $M=(\phi(k))_{k \in \mathbf{Z}}$. We claim that

$$
\begin{aligned}
\sup _{k \in \mathbb{Z}}\|\phi(k)\| & <\infty, \\
\sup _{k \in \mathbb{Z}}\left\|k^{l}\left(\Delta^{l} M\right)(k)\right\| & <\infty,
\end{aligned}
$$

for $l \in \mathbb{N}$. Indeed (4) is clearly true. We will only give the proof for (5) when $l=2$, the proof for the general case is similar.

First notice that when $4 h_{n} \leq k \leq 8 h_{n}-2$ for some $n \in \mathbb{N}$, or $k \leq 0$, then $\left(\Delta^{2} M\right)(k)=0$. While when $2 h_{n}-2<k<4 h_{n}$ for some $n \in \mathbb{N}$

$$
\begin{aligned}
\left(\Delta^{2} M\right)(k) & =\left(\phi_{n}(k+2)-2 \phi_{n}(k+1)+\phi_{n}(k)\right) N_{n} \\
& =\left(\phi_{1}\left(\frac{k+1}{h_{n}}+\frac{1}{h_{n}}\right)-2 \phi_{1}\left(\frac{k+1}{h_{n}}\right)+\phi_{1}\left(\frac{k+1}{h_{n}}-\frac{1}{h_{n}}\right)\right) N_{n} \\
& =\frac{1}{2 h_{n}^{2}}\left(\phi_{1}^{\prime \prime}\left(\eta_{1}\right)+\phi_{1}^{\prime \prime}\left(\eta_{2}\right)\right) N_{n}
\end{aligned}
$$

for some $\eta_{1}, \eta_{2} \in \mathbb{R}$. We deduce that

$$
\sup _{k \in \mathbb{Z}}\left\|k^{2}\left(\Delta^{2} M\right)(k)\right\| \leq \sup _{n \in \mathbb{N}} \frac{16 h_{n}^{2}}{4 h_{n}^{2}}\left\|N_{n}\right\| \sup _{x \in \mathbb{R}}\left|\phi_{1}^{\prime \prime}(x)\right| \leq 4 \sup _{n \in \mathbb{N}}\left\|N_{n}\right\| \sup _{x \in \mathbb{R}}\left|\phi_{1}^{\prime \prime}(x)\right| .
$$

Thus $M=(\phi(k))_{k \in \mathbb{Z}}$ is a periodic $L^{p}-L^{q}$-Fourier multiplier by assumption. Hence there exists $C>0$ such that for $n \in \mathbb{N}$ and $x_{1}, x_{2}, \ldots, x_{n} \in X$, we have

$$
\left\|\sum_{k} e_{k} \otimes \phi(k) x_{k}\right\|_{q} \leq C\left\|\sum_{k} e_{k} \otimes x_{k}\right\|_{p},
$$

and, in particular,

$$
\left\|\sum_{n \geq 1} e_{3 h_{n}} \otimes M_{k} x_{3 h_{n}}\right\|_{q} \leq C\left\|\sum_{n \geq 1} e_{3 h_{n}} \otimes x_{3 h_{n}}\right\|_{p} .
$$

By (3), this implies that the sequence $\left(M_{k}\right)_{k \geq 1}$ is $R$-bounded. It is easy to check that if each countable subset of $T$ is $R$-bounded then so is $T$. We deduce from this that each bounded subset in $\mathscr{L}(X)$ is actually $R$-bounded. By [3, Proposition 1.13], this implies that $X$ is isomorphic to a Hilbert space. 


\section{Multipliers on the line}

Let $X$ be a Banach space and consider the Banach space $L^{p}(\mathbb{R} ; X)$ for $1<p<\infty$. We denote by $\mathscr{D}(\mathbb{R} ; X)$ the space of all $X$-valued $C^{\infty}$-functions with compact support. $\mathscr{S}(\mathbb{R} ; X)$ will be the $X$-valued Schwartz space and we let $\mathscr{S}^{\prime}(\mathbb{R} ; X):=\mathscr{L}(\mathscr{S}(\mathbb{R}) ; X)$, where $\mathscr{S}(\mathbb{R})$ denotes the $\mathbb{C}$-valued Schwartz space. Let $Y$ be another Banach space. Then given $M \in L_{\text {loc }}^{1}(\mathbb{R} ; \mathscr{L}(X, Y))$, we may define an operator $T: \mathscr{F}-1 \mathscr{D}(\mathbb{R} ; X) \rightarrow$ $\mathscr{S}^{\prime}(\mathbb{R} ; Y)$ by means of

$$
T \phi:=\mathscr{F}^{-1} M \mathscr{F} \phi \quad \text { for all } \mathscr{F} \phi \in \mathscr{D}(\mathbb{R} ; X)
$$

where $\mathscr{F}$ denotes the Fourier transform. Since $\mathscr{F}^{-1} \mathscr{D}(\mathbb{R} ; X)$ is dense in $L^{p}(\mathbb{R} ; X)$, we see that $T$ is well defined on a dense subset of $L^{p}(\mathbb{R} ; X)$. We say that $M$ is an $L^{p}$-Fourier multiplier on $L^{p}(\mathbb{R} ; X)$ if $T$ can be extended to a bounded linear operator from $L^{p}(\mathbb{R} ; X)$ to $L^{p}(\mathbb{R} ; Y)$.

The classical Mikhlin Fourier multiplier theorem has been extended to the operatorvalued case by Weis. Let $X$ and $Y$ be UMD spaces, $1<p<\infty$ and let $M \in$ $C^{1}(\mathbb{R} \backslash\{0\}, \mathscr{L}(X, Y))$. If both $\{M(x): x \neq 0\}$ and $\left\{x M^{\prime}(x): x \neq 0\right\}$ are $R$-bounded, then $M$ defines a $L^{p}$-Fourier multiplier on $L^{p}(\mathbb{R} ; X)[18]$.

The following result shows that one cannot replace $R$-boundedness in the operatorvalued Mikhlin theorem above by boundedness in operator norm unless the underlying Banach space is isomorphic to a Hilbert space.

THEOREM 3. Let $X$ be a Banach space. Then the following assertions are equivalent:

(i) $X$ is isomorphic to a Hilbert space.

(ii) For some $1<p<\infty$, each function $M \in C^{\infty}(\mathbb{R} ; \mathscr{L}(X))$ satisfying

(a) $M(x)=0$ for $x \leq 0$,

(b) $\sup _{x \in \mathbb{R}}\|M(x)\|<\infty$,

(c) $\sup _{x \in \mathbb{R}}(1+|x|)^{l}\left\|M^{(l)}(x)\right\|<\infty$ for $l \in \mathbb{N}$,

defines an $L^{p}$-Fourier multiplier on $L^{p}(\mathbb{R} ; X)$.

(iii) For all $1<p<\infty$, each function $M \in C^{1}(\mathbb{R} \backslash\{0\} ; \mathscr{L}(X))$ satisfying the conditions

(a) $\sup _{x \neq 0}\|M(x)\|<\infty$,

(b) $\sup _{x \neq 0}\left\|x M^{\prime}(x)\right\|<\infty$,

defines an $L^{p}$-Fourier multiplier on $L^{p}(\mathbb{R} ; X)$.

Proof. (i) $\Rightarrow$ (iii). Assume that $X$ is isomorphic to a Hilbert space. Then considering an orthonormal basis one easily verifies that each bounded subset in $\mathscr{L}(X)$ is 
actually $R$-bounded, so the result follows from the operator-valued Mikhlin Fourier multiplier theorem of Weis [18].

(iii) $\Rightarrow$ (ii) is trivial.

(ii) $\Rightarrow$ (i). Assume (ii) holds. Let $\left(M_{k}\right)_{k \geq 0} \subset \mathscr{L}(X)$ be a bounded sequence and let $\phi \in \mathscr{D}(\mathbb{R})$ satisfying $\operatorname{supp}(\phi) \subset[1,2]$, $\sup _{x \in \mathbb{R}}|\phi(x)|=1$ and $\phi(3 / 2)=1$. Define $M \in C^{\infty}(\mathbb{R} ; \mathscr{L}(X))$ by

$$
M(x)= \begin{cases}0 & \text { if } x \leq 1 \\ \phi\left(2^{-k} x\right) M_{k} & \text { if } 2^{k} \leq x<2^{k+1} \text { for some } k \geq 0 .\end{cases}
$$

Then $\sup _{x \in \mathbb{R}}\|M(x)\|=\sup _{k \geq 0}\left\|M_{k}\right\|<\infty$ and for $l \in \mathbb{N}$,

$$
\begin{aligned}
\sup _{x \in \mathbb{R}}(1 & +|x|)^{l}\left\|M^{(l)}(x)\right\| \\
\leq & 2^{m}\left(\sup _{x \in \mathbb{R}}\left|x^{l} \phi^{(l)}(x)\right| \sup _{k \geq 0}\left\|M_{k}\right\|+\sup _{x \in \mathbb{R}}\left|\phi^{(l)}(x)\right| \sup _{k \geq 0} 2^{-l k}\left\|M_{k}\right\|\right)<\infty .
\end{aligned}
$$

So $M$ is an $L^{p}$-Fourier multiplier on $L^{p}(\mathbb{R} ; X)$ by assumption. By [9, Proposition 1] this implies that the set $\{M(x): x \in \mathbb{R}\}$ is $R$-bounded. In particular, the sequence $\left(M_{k}\right)_{k \geq 0}$ is $R$-bounded. We deduce from this that each bounded subset in $\mathscr{L}(X)$ is $R$-bounded, by [3, Proposition 1.13] $X$ is isomorphic to a Hilbert space.

\section{Multipliers on $\mathbb{Z}$}

Let $X, Y$ be Banach spaces and consider the Banach space $\ell^{p}(\mathbb{Z} ; X)$ for $1<$ $p<\infty$. Let $\mathbb{T}=\left\{e^{i t}: 0 \leq t<2 \pi\right\}$ be the torus. We consider the dense subspace $P$ of $\ell^{p}(\mathbb{Z} ; X)$ consisting of all elements having a finite support. Then for $f=\left(f_{n}\right)_{n \in \mathbb{Z}} \in P$, the Fourier transform of $f$ is a function on $[-\pi, \pi]$ defined by $(\mathscr{F} f)(t)=\sum_{n \in \mathbb{Z}} f_{n} e^{i n t}$. Let $M \in L^{\infty}(-\pi, \pi ; \mathscr{L}(X, Y))$. Then the function $M \mathscr{F} f$ is in $L^{\infty}(-\pi, \pi ; Y)$, where $\mathscr{F}^{-1}$ denotes the inverse Fourier transform. We deduce that that $T f:=\mathscr{F}^{-1}(M \mathscr{F} f) \in c_{0}(\mathbb{Z} ; Y)$ makes sense. We say that $M$ is an $L^{p}$ Fourier multiplier on $\ell^{p}(\mathbb{Z} ; X)$ if the mapping $T$ can be extended to a bounded linear operator from $\ell^{p}(\mathbb{Z} ; X)$ to $\ell^{p}(\mathbb{Z} ; Y)$.

The classical Mikhlin Fourier multiplier theorem on $\ell^{p}(\mathbb{Z})$ has been extended to the operator-valued case by Blunck. Let $1<p<\infty, X$ be a UMD space, let $M \in$ $C^{1}((-\pi, 0) \cup(0, \pi) ; \mathscr{L}(X))$ be such that both $\{M(t): t \in(-\pi, 0) \cup(0, \pi)\}$ and $\left\{\left(e^{i t}-\right.\right.$ 1) $\left.\left(e^{i t}+1\right) M^{\prime}(t): t \in(-\pi, 0) \cup(0, \pi)\right\}$ are $R$-bounded. Then $M$ is an $L^{p}$-Fourier multiplier on $\ell^{p}(\mathbb{Z} ; X)$ [5]. In particular, each $M \in C^{1}([-\pi, 0) \cup(0, \pi] ; \mathscr{L}(X))$ such that both $\{M(t): t \neq 0\}$ and $\left\{t M^{\prime}(t): t \neq 0\right\}$ are $R$-bounded, defines an $L^{p}$-Fourier multiplier on $\ell^{p}(\mathbb{Z} ; X)$. Blunck has also established the $R$-boundedness of 
$L^{p}$-Fourier multipliers on $\ell^{p}(\mathbb{Z} ; X)$ : when $M$ is an $L^{p}$-Fourier multiplier on $\ell^{p}(\mathbb{Z} ; X)$, then $\{M(t): t$ is a Lebesgue point of $M\}$ is $R$-bounded.

The following result shows that one cannot replace the $R$-boundedness in Blunck's result by the boundedness in operator norm unless the underlying Banach space is isomorphic to a Hilbert space. As the proof is similar to that of Theorem 3, we omit it.

THEOREM 4. Let $X$ be a Banach space. Then the following assertions are equivalent:

(i) $X$ is isomorphic to a Hilbert space.

(ii) For some $1<p<\infty$, each function $M \in C^{\infty}([-\pi, \pi]$; $\mathscr{L}(X))$ satisfying

(a) $\sup _{x \in[-\pi, \pi]}\|M(x)\|<\infty$,

(b) $\sup _{x \in[-\pi, \pi]}|x|^{l}\left\|M^{(l)}(x)\right\|<\infty$ for $l \in \mathbb{N}$,

(c) $M(x)=0$ for $x \leq 0$,

defines an $L^{p}$-Fourier multiplier on $\ell^{p}(\mathbb{Z} ; X)$.

(iii) For all $1<p<\infty$, each function $M \in C^{1}([-\pi, 0) \cup(0, \pi] ; \mathscr{L}(X))$ satisfying

(a) $\sup _{x \neq 0}\|M(x)\|<\infty$,

(b) $\sup _{x \neq 0}\left\|x M^{\prime}(x)\right\|<\infty$,

defines an $L^{p}$-Fourier multiplier on $\ell^{p}(\mathbb{Z} ; X)$.

\section{Concluding remarks}

(a) One can actually show by using [3, Theorem 1.3] and the same argument as in the proof of Theorem 1, that when $X$ and $Y$ are UMD-spaces, then the assertions (ii) and (iii) in Theorem 1 are still equivalent for sequences in $\mathscr{L}(X, Y)$. Similarly, using [18, Theorem 3.4] (respectively, [5, Theorem 1.3]) one can show that when $X$ and $Y$ are UMD-spaces, the assertions (ii) and (iii) in Theorem 3 (respectively, Theorem 4) are still equivalent for functions with values in $\mathscr{L}(X, Y)$. Furthermore, these assertions are equivalent to $X$ having cotype 2 and $Y$ having type 2 . This contains our Theorem 1 , Theorem 3 and Theorem 4 by a result of Kwapien [14, pages 73-74], saying that a Banach space $X$ is isomorphic to a Hilbert space if and only if $X$ is of cotype 2 and of type 2.

(b) A restricted version of our results follows from the recent work of Kalton and Lancien on the maximal regularity problem [12]. In particular, the counterexample constructed in [12] can be used to show that the equivalences in Theorem 1 and Theorem 3 are true within the class of UMD Banach spaces which have an unconditional basis.

(c) In contrast to the $L^{p}$-spaces case, the situation for Hölder continuous function spaces is quite different. It has been shown that the operator-valued Marcinkiewicz 
(respectively, Mikhlin) Fourier multiplier theorem holds true on $C_{\text {per }}^{\alpha}([0,2 \pi] ; X)$ (respectively, $C^{\alpha}(\mathbb{R} ; X)$ ) for every Banach space $X$ and $0<\alpha<1$ and for each sequence $M=\left(M_{k}\right)_{k \in \mathbb{Z}} \subset \mathscr{L}(X)$ satisfying a second order condition:

$$
\sup _{k \in \mathbb{Z}}\left\|M_{k}\right\|+\sup _{k \in \mathbb{Z}}\left\|k\left(\Delta^{1} M\right)(k)\right\|+\sup _{k \in \mathbb{Z}}\left\|k^{2}\left(\Delta^{2} M\right)(k)\right\|<\infty
$$

(respectively, each function $M \in C^{2}(\mathbb{R} \backslash\{0\} ; \mathscr{L}(X))$ satisfying a second order condition: $\sup _{x \neq 0}\|M(x)\|+\sup _{x \neq 0}\left\|x M^{\prime}(x)\right\|+\sup _{x \neq 0}\left\|x^{2} M^{\prime \prime}(x)\right\|<\infty$ ) (see Amann [1] and [2]). Here $C_{\text {per }}^{\alpha}([0,2 \pi] ; X)$ denotes the space of all functions in $C^{\alpha}(\mathbb{R}, X)$ which are $2 \pi$-periodic. If the Banach space has a non-trivial type, then even the Marcinkiewicz condition of order 1 suffices (see [2] and [11]).

(d) Periodic $L^{p}$-Fourier multipliers (respectively, $L^{p}$-Fourier multipliers on $L^{p}(\mathbb{R}$; $X)$ ) of the form $M=\left(m_{k} I\right)_{k \in \mathbb{Z}}$, where $m_{k} \in \mathbb{C}$ for $k \in \mathbb{Z}$ (respectively, $M=f I$, where $f \in C^{1}(\mathbb{B} \backslash\{0\})$ ) on $L^{p}(0,2 \pi ; X)$ (respectively, on $L^{p}(\mathbb{R} ; X)$ ) have been studied by Zimmermann [19], where $I$ denotes the identity operator on $X$. Actually Zimmermann's results follow from the operator-valued Marcinkiewicz (respectively, Mikhlin) Fourier multiplier theorem established in [3] (respectively, in [18]) as each subset $M \subset \mathscr{L}(X)$ of the form $M=\{\lambda I: \lambda \in \Omega\}$ is $R$-bounded whenever $\Omega \subset \mathbb{C}$ is bounded. Zimmermann's results together with a result of Burkholder [7] show that the scalar-valued Marcinkiewicz (respectively, Mikhlin) Fourier multiplier theorem holds true for $L^{p}(0,2 \pi ; X)$ (respectively, $L^{p}(\mathbb{R} ; X)$ ) for some $1<p<\infty$ if and only if $X$ is a UMD space. A similar result characterizing UMD spaces via a scalar-valued Fourier multiplier theorem on $\ell^{p}(\mathbb{Z} ; X)$ can be established based on results in [4].

(e) It is remarkable that in all three cases we consider here (Theorem 1, Theorem 3 and Theorem 4), the sequence $\left(M_{k}\right)_{k \in \mathbb{Z}}$ (or the function $M$ ) satisfying the Marcinkiewicz condition (of order $l$ ) without being a Fourier multiplier consists of operators of rank 1 (see [3, Proposition 1.13.]).

\section{References}

[1] H. Amann, 'Operator-valued Fourier multipliers, vector-valued Besov spaces, and applications', Math. Nachr. 186 (1997), 5-56.

[2] W. Arendt, C. Batty and S. Bu, 'Fourier multipliers for Hölder continuous functions and maximal regularity', Studia Math. 160 (2004), 23-51.

[3] W. Arendt and S. Bu, 'The operator-valued Marcinkiewicz multiplier theorem and maximal regularity', Math. Z. 240 (2002), 311-343.

[4] E. Berkson and T. A. Gillespie, 'Spectral decompositions and harmonic analysis on UMD-spaces', Studia Math. 112 (1994), 13-49.

[5] S. Blunck, 'Maximal regularity of discrete and continuous time evolution equations', Studia Math. 146 (2001), 157-163. 
[6] J. Bourgain, 'Vector-valued singular integrals and the $H^{1}-B M O$ duality', in: Probability theory and harmonic analysis (ed. D. Burkholder) (Dekker, New York, 1986) pp. 1-19.

[7] D. Burkholder, 'A geometric condition that implies the existence of certain singular integrals of Banach-space-valued functions', in: Proc. of Conf. on Harmonic Analysis in Honor of Antoni Zygmund, Chicago 1981 (Wadsworth Publishers, Belmont, CA, 1983) pp. 270-286.

[8] Ph. Clément, B. de Pagter, F. A. Sukochev and M. Witvliet, 'Schauder decomposition and multiplier theorems', Studia Math. 138 (2000), 135-163.

[9] $\mathrm{Ph}$. Clément and J. Prüss, 'An operator-valued transference principle and maximal regularity on vector-valued $L_{p}$-spaces', in: Evolution equations and their applications in physics and life sciences (eds. Lumer and L. Weis), Lecture Notes in Pure and Appl. Math. 215 (Dekker, New York, 2000) pp. 67-87.

[10] R. Denk, M. Hieber and J. Prüss, ' $R$-boundedness, Fourier multipliers and problems of elliptic and parabolic type', Mem. Amer. Math. Soc. 166 (Amer. Math. Soc., Providence, 2003), p. 788.

[11] M. Girardi and L. Weis, 'Operator-valued Fourier multiplier theorems on Besov spaces', Math. Nachr. 251 (2003), 34-51.

[12] N. J. Kalton and G. Lancien, 'A solution of the $L^{p}$-maximal regularity', Math. Z. 235 (2000), 559-568.

[13] $\longrightarrow$, ' $L^{p}$-maximal regularity on Banach spaces with a Schauder basis', Arch. Math. (Basel) 78 (2002), 397-408.

[14] J. Lindenstrauss and L. Tzafriri, Classical Banach spaces II (Springer, Berlin, 1979).

[15] G. Pisier, Les inégalités de Khintchine-Kahane d'après C. Borell, Séminaire sur la géometrie des espaces de Banach 7 (Ecole Polytechnique Paris, 1977).

[16] W. Rudin, Fourier analysis on groups (Wiley, New York, 1990).

[17] Z. Strkalj and L. Weis, 'On operator-valued Fourier multiplier theorems', preprint, 1999.

[18] L. Weis, 'Operator-valued Fourier multiplier theorems and maximal $L_{p}$-regularity', Math. Ann. 319 (2001), 735-758.

[19] F. Zimmermann, 'On vector-valued Fourier multiplier theorems', Studia Math. 93 (1989), 201-222.

Abteilung Angewandte Analysis

Universität Ulm

89069 Ulm

Germany

e-mail: arendt@mathematik.uni-ulm.de
Department of Mathematical Science

University of Tsinghua

Beijing 100084

China

e-mail: sbu@math.tsinghua.edu.cn 